\title{
Barriers in the implementation of isoniazid preventive therapy for people living with HIV in Northern Ethiopia: a mixed quantitative and qualitative study
}

${\text { Gebrehiwot Teklay }{ }^{1 *}, \text { Tsigemariam Teklu², Befikadu Legesse }^{1,3}, \text { Kiros Tedla }^{4} \text { and Eveline Klinkenberg }}^{5,6}$

\begin{abstract}
Background: Isoniazid preventive therapy is a key public health intervention for the prevention of tuberculosis disease among people living with HIV. Despite the confirmed efficacy of isoniazid preventive therapy and global recommendations existing for decades, its implementation remains limited. In resource constrained settings, few have investigated why isoniazid preventive therapy is not implemented on full scale. This study was designed to investigate the level of isoniazid preventive therapy implementation and reasons for suboptimal implementation in Tigray region of Ethiopia.

Methods: A review of patient records combined with a qualitative study using in-depth interviews and focus group discussions was conducted in 11 hospitals providing isoniazid preventive therapy in the Tigray Region. The study participants were health providers working in the HIV clinics of the 11 hospitals in the province. Health providers were interviewed about their experience of providing isoniazid preventive therapy and challenges faced during its implementation. All conversations were audio-recorded. Record review of 16,443 HIV patients registered for care in these hospitals between September 2011 and April 2014 was done to determine isoniazid preventive therapy utilization. Data were collected from April to August 2014.
\end{abstract}

Results: Fifty health providers participated in the study. Overall isoniazid preventive therapy coverage of the region was estimated to be $20 \%$. Isoniazid stock out, fear of creating isoniazid resistance, problems in patient acceptance, and lack of commitment of health managers to scale up the program were indicated by health providers as the main barriers hindering implementation of isoniazid preventive therapy.

Conclusion: Implementation of isoniazid preventive therapy in Tigray region of Ethiopia had low coverage. Frequent interruption of isoniazid supplies raises the concern of interrupted therapy resulting in creation of isoniazid resistance. Health managers, drug suppliers and partners working in HIV and tuberculosis programs should be committed to ensure an uninterrupted supply of isoniazid and full scale implementation of isoniazid preventive therapy to eligible people living with HIV.

Keywords: Challenges, Isoniazid stock out, IPT coverage, Tigray

Abbreviations: $\mathrm{CD} 4$, Cluster differentiation; $\mathrm{Cl}$, Confidence interval; COREQ, Consolidated criteria for reporting qualitative research; ERC, Ethics Review Committee; HIV, Human immunodeficiency virus; IPT, Isoniazid preventive therapy; N, Number; PFSA, Pharmaceuticals Fund and Supply Agency; PLHIV, People living with HIV; SD, Standard deviation; TB, Tuberculosis; TRAC/FMOH, Tuberculosis Research Advisory Committee/Federal Ministry of Health; WHO, World Health Organization

\footnotetext{
* Correspondence: tgebrehiwet@gmail.com

'Department of Pharmacy, College of Health Sciences, Mekelle University,

Mekelle, Ethiopia

Full list of author information is available at the end of the article
} 


\section{Background}

Tuberculosis (TB) preventative therapy for people living with HIV (PLHIV) is necessary and an important public health intervention especially in developing countries where both TB and HIV are serious problems and known to be synergistic and bidirectional. The World Health Organization (WHO) recommends the use of at least six months Isoniazid preventive therapy (IPT) for PLHIV without active TB in countries with a high burden of TB infection [1]. Ethiopia is classified as one of the world's high TB burden, high multi-drug resistantTB burden and high TB-HIV burden country by WHO [2]. IPT implementation in the country started in 2005 [3]. Per national guidelines, all PLHIV should be screened for active TB disease using the WHO foursymptom based screening (current cough, fever, night sweats or weight loss). If no evidence of active TB is found, IPT should be provided [1, 3].

Many studies demonstrated the benefit of IPT among PLHIV [3-11]. A Cochrane review of 12 randomized controlled trials revealed that IPT lowers the incidence of active TB compared to placebo (relative risk 0.68, $95 \%$ confidence interval (CI) 0.54 to 0.85 ) [3]. Recent studies in Ethiopia reported a reduction of about $50 \%$ in tuberculosis incidence in patients using IPT $[5,6]$. Despite the confirmed efficacy of IPT and existing global recommendations and policy by the WHO since 2004, its implementation remains limited. Globally, the number of PLHIV who were treated with IPT reached 933 000 in 2014, an increase of about $60 \%$ compared with 2013 [2, 12]. Nonetheless, $77 \%$ of countries did not report provision of IPT as part of HIV care in 2014.

The implementation guideline for TB/HIV collaborative activities in Ethiopia states that IPT can be given to PLHIV after screening for the absence of four TB suggestive symptoms (current cough, fever, night sweats and weight loss) and any contraindications [3]. To those for whom active TB is excluded, a daily, selfadministered dose of isoniazid is given for a period of six months at a dose of $5 \mathrm{mg} / \mathrm{kg}$ for children and $300 \mathrm{mg} /$ day for adults. IPT can be initiated before or after the initiation of antiretroviral therapy. In Ethiopia, the number of PLHIV newly enrolled in care who initiated IPT in 2014 was 10,385 (excluding the missing data for 3 of Ethiopia's 11 regions) [12]. A study by Cowan et al. [13] reported low IPT implementation in the Tigray region of Ethiopia.

Different reasons have been pointed out for the low uptake of IPT implementation globally. These are amongst others, related to health service providers' knowledge and perception about IPT provision, inadequate application of the symptom screening tool (intensified TB case finding) to rule out active TB, inadequate patient adherence to IPT and fear of amplifying resistance to isoniazid through IPT [12-17]. In Ethiopia limited evidence is available regarding the status of and barriers to IPT implementation. As IPT coverage is known to be limited, it would be important to determine the actual level of IPT implementation and the reasons for its low uptake.

\section{Methods}

This study was conducted in Tigray region located in northern Ethiopia. This study setting was selected to further investigate the reports by Cowan et al. [13] which indicated low uptake of IPT implementation in the region. As per the 2014 health status report, the region had 102 health facilities providing care to PLHIV [18]. In the region, IPT was initiated late in the year 2010. Eleven public hospitals, offering IPT for at least three or more years were included in this study. A further three newly established hospitals as well as all the health centers in the region were not included as these health facilities were not providing IPT yet as per unpublished reports obtained from the Tigray regional health bureau.

A mixed approach of quantitative and qualitative study designs was employed. The quantitative section aimed to determine IPT coverage of the region while in-depth interviews and focus group discussions were used to investigate barriers for health workers to implement IPT. The quantitative data were collected by using a structured data collection form and review of patient records. Information on availability of isoniazid, functionality of $\mathrm{x}$-ray machine, stock out of reagents and slides required for the diagnosis of TB was collected from each hospital. Information on the number of PLHIV registered in HIV care and provided with IPT between September 2011 and April 2014 was collected from IPT registries of each hospital. IPT coverage was determined as percentage of PLHIV started on IPT among all PLHIV registered in HIV care.

Health providers (physicians, nurses, health officers, pharmacy workers and laboratory staff) were the interviewees and all participated in the focus group discussions. Health providers who were present during the time of the visit of the field team to the 11 hospitals and having at least 5 months working experience in HIV care were included in the qualitative study. The study participants were approached with the assistance of the trained study focal person in each hospital, selected and trained to collect data. The focal persons were recruited among nurses and pharmacists working in the 11 hospitals but not in the HIV clinic. The focal persons were trained as data collectors on how to approach study participants, how to ask for informed consent, conduct interviews and focus groups, collect quantitative data from patient records while maintaining the confidentiality of the collected data. The in-depth-interviews and focus groups 
were conducted in a separate room in each hospital close to the HIV clinic to ensure privacy. Each interviewee was first asked individually about his/her experience of providing IPT and challenges faced in its implementation. This was then followed by a focus group discussion to identify common barriers hindering implementation of IPT. Each in-depth interview took about 25 min while focus groups had an average duration of about 35 min till saturation was reached. The focus group discussions were moderated by the investigators. All the planned in-depth interviews in the 11 hospitals (three to five interviews in each hospital) were conducted. A total of 8 representative focus groups were conducted (one focus group per hospital) till saturation was reached. Data were collected between April and August, 2014.

Data from both quantitative and qualitative parts were analyzed by the investigators. The quantitative data were analyzed using SPSS for windows version 20 statistical software. Data was checked for completeness and accuracy through proofreading while entering the data into the software. Descriptive statistics (frequencies and percentage) were employed and data were visualized using tables and bar graphs. Audio-recorded data from the indepth interviews and focus groups were transcribed using verbatim and translated into English. Qualitative data verification for accuracy and completeness was done through reading and re-reading by different investigators to ensure all recorded information and variations were identified. After transcription, codes were developed by two investigators based on the original terms used, and were matched. The transcripts and notes were analyzed thematically by categorizing them in line with the specific objectives (providers, patient, and resource requirement related barriers for implementation of IPT). The codes were presented, discussed and checked within the research team. Tentative categories and sub-categories were created from the clustered codes, and subsequently main themes emerged based on the patterns and relationships between the categories. Main themes were illustrated with representative quotations.

\section{Results}

Fifty health providers participated in the qualitative study. The mean age $( \pm S D)$ of health providers was 31 $( \pm 6)$ years. Twenty four $(48 \%)$ of the participants were nurses. Thirty $(60 \%)$ of health providers had more than two years working experience in HIV care (Table 1).

All the 11 hospitals reported having a functional chest $\mathrm{x}$-ray machine. Four hospitals $(36.4 \%)$ reported at least once a stock out in the past one year of microscopic reagents and/or slides for the diagnosis of TB. In the year 2013, shortage of adult and pediatric dose of isoniazid
Table 1 Demographic characteristics of health providers interviewed at 11 HIV clinics of Tigray, Northern Ethiopia, 2014

\begin{tabular}{|c|c|c|c|}
\hline \multicolumn{2}{|l|}{ Demographic characteristics } & \multicolumn{2}{|c|}{ Health providers $(N=50)$} \\
\hline & & $\mathrm{N}$ & $\%$ \\
\hline \multirow[t]{2}{*}{ Age (yrs) } & $<30$ & 25 & 50.0 \\
\hline & $30-60$ & 25 & 50.0 \\
\hline \multirow[t]{2}{*}{ Sex } & Female & 21 & 42.0 \\
\hline & Male & 29 & 58.0 \\
\hline \multirow[t]{5}{*}{ Profession } & Nurse & 24 & 48.0 \\
\hline & Health officer & 7 & 14.0 \\
\hline & $\begin{array}{l}\text { Medical } \\
\text { doctor }\end{array}$ & 4 & 8.0 \\
\hline & Pharmacy & 10 & 20.0 \\
\hline & Laboratory & 5 & 10.0 \\
\hline \multirow{2}{*}{$\begin{array}{l}\text { Work experience in HIV care } \\
\text { (yrs) }\end{array}$} & $<2$ & 20 & 40.0 \\
\hline & $\geq 2$ & 30 & 60.0 \\
\hline
\end{tabular}

was reported in seven and four of the 11 hospitals respectively. This resulted in interruption of IPT among PLHIV already on IPT and limited initiation of IPT for new PLHIV.

Out of the 16,443 PLHIV registered in the HIV clinics between September 2011 and April 2014, 3230 (19.6 \%) had initiated IPT and $68 \%$ of them had documentation on completion of six month IPT (Fig. 1). Despite the adoption of IPT policy in the country since 2005, health facilities included in this study started implementation in the years 2010 to 2012. IPT coverage varied between hospitals; two hospitals had high IPT coverage (of $47.0 \%$ and $36.2 \%$ ) while two other hospitals had a much lower coverage (of $8.1 \%$ and $9.2 \%$ ). Also, four of the 11 hospitals were not providing IPT at the time of the study due to isoniazid stock outs. The shortage had been experienced since about two months; some PLHIV already on IPT were forced to discontinue their treatment. Besides interrupting treatment for those on IPT, it limited initiation of IPT for new PLHIV. Low IPT coverage was more seen among the facilities that started IPT implementation late, in 2012 (Table 2).

\section{Challenges in IPT implementation Irregularity of isoniazid supply and fear of drug resistance} Although most providers believed that IPT can be effective, multiple factors had impeded implementation of IPT in the region. Most concerns raised were about the unreliable provision of isoniazid causing fear of isoniazid resistance. This was raised as a concern by almost all health providers during all focus groups as illustrated by the following quotes:

'One time they (regional administrators) say go ahead, next time shortage happens after patients take for two 


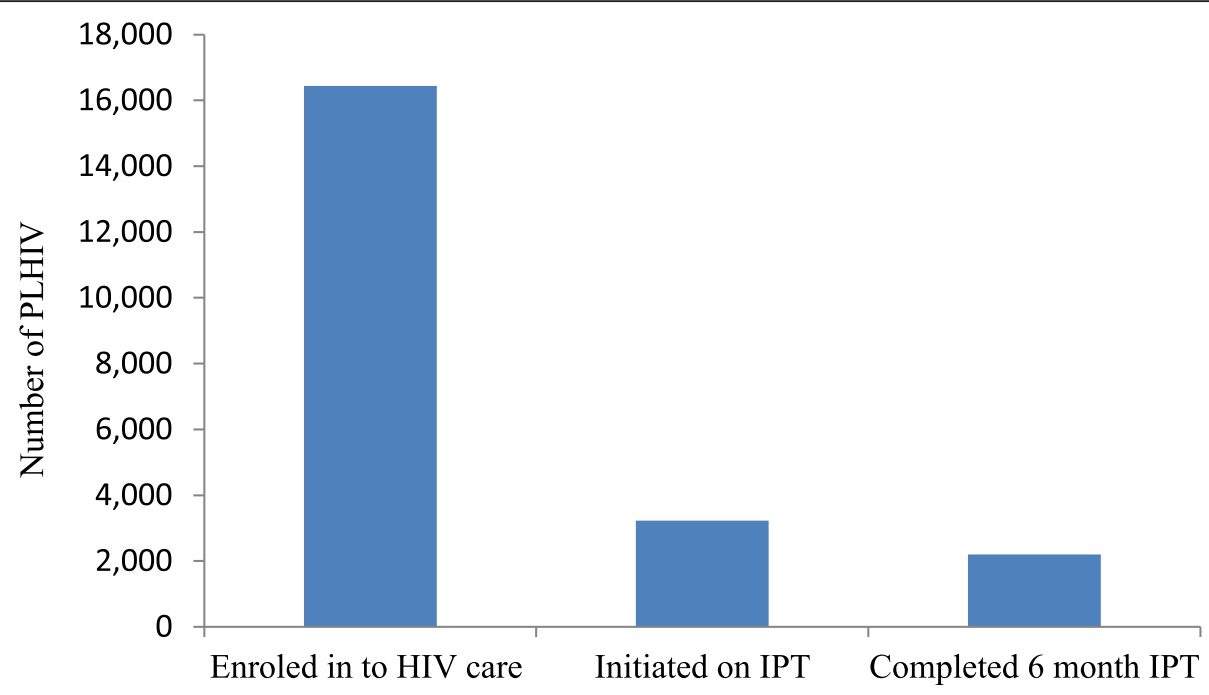

Fig. 1 Number of PLHIV provided with IPT in 11 hospitals of Tigray, Northern Ethiopia, September 2011- April 2014

or three months. Many patients are stopping it without completion. We can't be sure of the continuity of supply that is why we usually hesitate to initiate new ones' -health officer

One participant emotionally said 'I don't know! Are we helping or hurting our patients? There is irregular supply of isoniazid. It was the first phase patients who completed 6 month treatment. The others had interruptions after taking two or three months. This may lead to isoniazid resistance' -doctor

'...despite the current availability of sufficient isoniazid, there are some reservations in initiating IPT for new patients due to fear of future stock outs' - pharmacist
'Since I started working in this hospital, I had never provided IPT. Either there is no medication or it's insufficient to our clients. We have strong fear of resistance development. Stock out is a common problem in our hospital' -health officer

'...we start today the interruption follows tomorrow. I believe the risk of resistance may outweigh its benefit. Our patients are also losing trust in us. When we told them (the patients) the medication is not available, they feel as they might be exposed to danger' -nurse

'It is not easy to identify those patients who need IPT. There could be missed opportunities. Providing this

Table 2 IPT start year and coverage of 11 hospitals of Tigray, Northern Ethiopia, September 2011-April 2014

\begin{tabular}{|c|c|c|c|c|c|}
\hline $\begin{array}{l}\text { Health } \\
\text { facility }\end{array}$ & $\begin{array}{l}\text { IPT start } \\
\text { year }\end{array}$ & $\begin{array}{l}\text { Number of PLHIV } \\
\text { registered }\end{array}$ & $\begin{array}{l}\text { Number of PLHIV on IPT at the time } \\
\text { of study }\end{array}$ & $\begin{array}{l}\text { Total number of PLHIV provided IPT } 2011 \\
\text { to } 2014\end{array}$ & $\begin{array}{l}\text { IPT coverage } \\
(\%)^{\mathrm{a}}\end{array}$ \\
\hline Hospital 1 & 2012 & 1734 & 0 & 159 & 9.2 \\
\hline Hospital 2 & 2012 & 1386 & 75 & 201 & 14.5 \\
\hline Hospital 3 & 2012 & 1320 & 58 & 159 & 12.0 \\
\hline Hospital 4 & 2012 & 1094 & 19 & 515 & 47.0 \\
\hline Hospital 5 & 2010 & 3229 & 0 & 634 & 19.6 \\
\hline Hospital 6 & 2011 & 1460 & 43 & 529 & 36.2 \\
\hline Hospital 7 & 2012 & 1327 & 45 & 108 & 8.1 \\
\hline Hospital 8 & 2012 & 954 & 0 & 142 & 14.9 \\
\hline Hospital 9 & 2011 & 776 & 6 & 186 & 24.0 \\
\hline Hospital 10 & 2012 & 1843 & 60 & 188 & 10.2 \\
\hline Hospital 11 & 2011 & 1320 & 0 & 409 & 31.0 \\
\hline Total & & 16,443 & 306 & 3,230 & 19.6 \\
\hline
\end{tabular}

PLHIV people living with HIV, IPT isoniazid preventive therapy

a IPT coverage was calculated by dividing the number of PLHIV registered in a particular health facility to the total number of PLHIV provided IPT 
single drug on top of the stock outs, obviously

resistance may develop' -doctor

'We sent drug susceptibility test for patients with presumed multi-drug resistant TB and they become isoniazid resistant. I always question if the isoniazid we are providing for prophylaxis is contributing to such resistance. We hesitate in initiation. Unless it's supported by data, it's difficult to say IPT is being beneficial ... -health officer

'Previously there were problems of awareness in most of us (exposure of patients to a single drug isoniazid may lead to resistance). But currently there is no such fear as far as we are treating latent TB. And the problems of on-off supply are currently corrected' -pharmacist

\section{Policy implementation gaps}

There are gaps between having the IPT policy in place and the actual implementation. The policy for IPT among PLHIV implementation was adopted nationally and implementation started in Ethiopia since 2005; however, the participants report that the policy was only implemented in Tigray region since 2011 due to fear of amplifying resistance to isoniazid through IPT. Health authorities' concern regarding isoniazid resistance in the region still exists due to irregularities in the drug supply. Not all HIV clinics in the region were permitted by the regional health authorities to offer IPT. At the time of this study, health centers providing antiretroviral therapy were not permitted to offer IPT, IPT was only being offered in hospitals. Lack of commitment of the health authorities and drug supply irregularities were raised by the service providers as reasons for the gap between having a policy and actual implementation. Health service providers also reported poor monitoring and lack of supervision of the IPT program by higher managers.

The following quotes illustrate the above interpretation.

IPT is a sensitive issue in this region because one time the region was refusing its use and was criticized as the only region not implementing. One time I remember the regional health authorities refusing its use unless there is continuous supply from pharmaceutical fund and supply agency (PFSA) ...' -nurse

'We ask isoniazid every two months from PFSA. But most of the time they had stock outs. I think there is a problem in the system. Priority should be given to PLHIV...' -pharmacist

$\therefore I P T$ is not being considered as prime treatment for PLHIV as antiretroviral therapy and cotrimoxazole. This is mainly the problem of the government and suppliers. If we can convince our patients that IPT will help them and the government is committed to supply isoniazid, it is possible to prevent tuberculosis in our patients' -pharmacist

'Previously IPT was not being provided to our patients. Now we have started it. There are problems of commitment from responsible bodies. We ourselves didn't push others as such for its implementation. I believe we don't have a problem of supply, it's due to lack of giving attention for the program. There are problems of convincing its' importance to our patients. There are also problems of following the implementation by higher authorities. IPT should be considered as prime issue' -health officer

Drug side effect, pill burden and adherence related barriers Providers reported that the unavailability of the adult dose of isoniazid resulted in a pill burden for PLHIV as instead of one, three tablets of the pediatric doses were provided to adults. This was compromising overall adherence. Most providers indicated that they prescribed IPT based on the patients' willingness and they indicated patients' were unwilling to add isoniazid to the antiretroviral therapy due to fear of having many pills.

'Sometimes it's only the $100 \mathrm{mg}$ isoniazid lefts in our stock. Providing three tablets for an adult is resulting in pill burden. Patients complain having many medications, and some gastrointestinal irritations. Hence, some patients are not willing to take it' pharmacist

'We had two patients who developed TB while on IPT. Such cases could be either a missed one during screening or due to immune reactivation in a patient with a low CD4 count or it could be because of patient non-adherence...'-doctor

Side effects such as gastrointestinal irritation, peripheral neuropathy and hepatotoxicity were also mentioned as challenges in IPT implementation. Some of the side effects are preventable however unavailability of pyridoxine had contributed to the problem of neuropathy.

‘..there is also shortage of pyridoxine. This could lead to increased risk of peripheral neuropathy...' pharmacist

'We had patients who interrupted the treatment due to shortage. Even currently we are not providing it. Its implementation is poor. It's only initiation, we never see a patient completing it. There is a big problem in supply. There are also some patients who interrupted 
IPT due to side effects such as hepatotoxicity and peripheral neuropathy' -nurse

\section{Lack of training and guidelines}

Health providers also reported the absence of training opportunities as well as availability of guidelines with regards to IPT. They believed that training should be provided first before the introduction of a program and it should be continued thereafter after for new staffs.

'We did not take special training regarding IPT. We have been trained within the antiretroviral therapy package and we don't have special guideline...' -nurse

'Training and update on IPT are lacking. I had never seen a new guideline, which are important to make us more confident' -health officer

'Also we are not trained in this regard. It was by letter they (regional health authorities) told us to start IPT' -nurse

The main barriers to IPT implementation underpinning the current research are summarized in a conceptual framework (Fig. 2).

\section{Discussion}

Implementation of IPT in the Tigray region of Ethiopia was started late and coverage was limited despite the fact that the policy has been in place in the country since 2005 [3]. The barriers hindering implementation of the IPT program were predominantly related to isoniazid supply problems, patient acceptance, concerns about amplifying resistance to isoniazid through IPT, and lack of commitment of health managers to scale up its implementation.

In this study only about one fifth (20 \%) of PLHIV registered in care were provided with IPT. This is lower than the reported IPT coverage of around $30 \%$ for Addis Ababa in 2011 [19, 20]. Differences in access to isoniazid could have contributed to the lower coverage observed in the northern region of Tigray as supply could have been better in Addis Ababa, the capital city of Ethiopia. Another reason for this difference could be related to the denominator used. In our study, IPT coverage was determined with all PLHIV registered in the HIV clinics as denominator, whereas the Addis Ababa studies calculated the coverage with the eligible as denominator this excluding those illegible to receive IPT. Our study was based on retrospective data, and eligibility of IPT could not be determined from the available routine records for all PLHIV therefore we adopted the wider denominator. Despite this difference, both results still indicate sub optimal coverage as the WHO target level of $50 \%$ coverage [12] is not yet achieved.

The main barrier leading to sub-optimal implementation of IPT observed in this study was shortage of isoniazid. It seems that lack of effective systems to manage drug supply is impeding implementation of the policy. Shortage of essential drugs, such as drugs used for TB and HIV treatment and prevention has been reported as a problem in health facilities of Ethiopia [21-24].

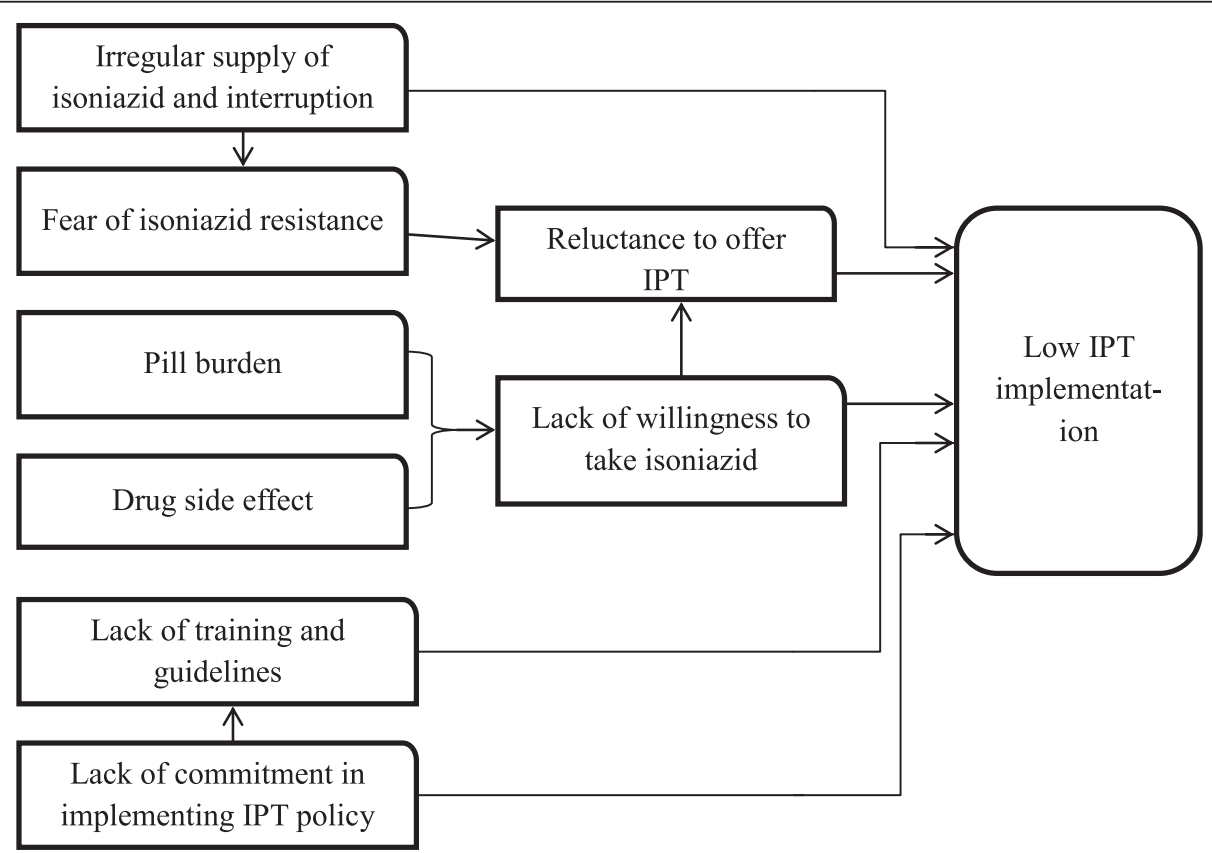

Fig. 2 Conceptual framework of barriers to implementation of IPT among PLHIV in Tigray, Northern Ethiopia, 2011-2014 
Non-availability of isoniazid was also observed by Getahun et al. [12] as a barrier to global IPT implementation. The supply problem of isoniazid could arise at the level of quantification, procurement, and/ or distribution. Participants in this study complained that they do not receive the quantities they requested. A study from Addis Ababa reported that only $47 \%$ of the health facilities received the full quantity of drugs they ordered [24].

The success of IPT implementation is mainly dependent on continuous resource mobilization, capacity building and monitoring. Health providers perceived that insufficient attention is being given to IPT by responsible bodies. Most of the hospitals in this study started implementing the IPT program just because they were told do so by higher officials. In some hospitals sensitization workshops, technical trainings, and operational guidelines were not provided. Health care staff also felt that there was no adequate monitoring and evaluation of the policy implementation. Similarly a study in Addis Ababa [16] reported that lack of reinforcement by health officials and stakeholders working in TB was affecting implementation of the IPT policy. A study in South Africa also reported lack of awareness on the benefits of IPT and the guidelines and recommendations by prescribers as primary barrier to IPT implementation [17]. Provision of a clear up-to-date policy and provision of trainings has been found to improve IPT implementation in other studies [25-27]. Trainings and good communication are vital for proper understanding of the policy and avoid ambiguities on IPT provision among health providers. This study did not include regional health managers, therefore their perspective is missing.

Lack of confidence in excluding active TB and fear of resistance development has been a matter of debate for many years $[28,29]$. The WHO strongly argues that concerns regarding the development of isoniazid resistance should not be a barrier for providing IPT [1] as the evidence disproves the development of isoniazidresistant TB after providing IPT to PLHIV [28, 30]. However, fear of isoniazid resistance is still a challenge to IPT implementation. In settings with limited diagnostic capacity, concerns about resistance may arise when use of chest X-ray is considered important to exclude active TB disease [31]. Concerns regarding amplifying resistance to isoniazid through IPT have been pointed out as challenge in several other studies [12, 17, 26, 32, 33].

Low completion rates of IPT were observed in this study. The main reasons could be related to shortage of isoniazid, poor patient adherence and side effects. Unavailability of adult isoniazid dose resulted in pill burden when providers instead issued three tablets of the pediatric doses to make up the adult dose. This resulted in unwillingness of patients to take IPT due to a concern of having too many tablets. And in those willing to take it, patient adherence was questionable due to the pill burden. A comparable study in Addis Ababa [16] also observed that poor patient adherence was a major barrier affecting the implementation of IPT. Concerns regarding pill burden and inadequate patient adherence were also raised in other studies [12, 15, 34]. In this study peripheral neuropathy and hepatotoxicity were reported as potential side effects related to IPT resulting in some patients discontinuing the treatment. Providers perceived that lack of pyridoxine in the hospitals increased the prevalence of peripheral neuropathy. A study by Durovni et al. [27] reported that $1.2 \%$ of persons initiating IPT discontinued therapy due to adverse reactions. However, the rate of hepatotoxicity associated with isoniazid was reported as low (0.001-0.15 \%) in several studies [9, 12, 33, 35].

The current study provides insight into the main reasons underlying health providers' reluctance to offer IPT like fear of resistance development due to frequent interruption of IPT, lack of clients' willingness to take isoniazid, and uncertainties on the future supply of the drug. Concerns about resistance development through IPT had been disproved in previous studies. However, these studies were done with full supply of IPT and without any interruption. In the current study the concern of frequent interruption of IPT supply was a big challenge for the IPT program. Future studies should focus on the risk of isoniazid resistance development through IPT in settings with irregular supplies and frequent interruption of IPT. Finally, this study determined the actual coverage of IPT but also gained insight in teasing out the underlying factors, some of them have been observed in other studies. Key factor here is the drug supply issue which is fixable and concrete actions can be taken.

\section{Conclusions}

Implementation of IPT in the Tigray region of Ethiopia was low with only one fifth of PLHIV covered. The main barrier mentioned hindering IPT implementation was irregular supply of isoniazid resulting in stock outs and interruption of IPT provision, creating fear among health care workers for development of drug resistance. Health managers, drug suppliers and partners working in HIV-TB programs should be committed to ensure an uninterrupted isoniazid supply for the success of IPT implementation.

\section{Additional file}

Additional file 1: Dataset. (XLSX $26 \mathrm{~kb}$ ) 


\section{Acknowledgments}

The research team is glad to thank the USAID/TB CARE I for the financial support. We thank the Tuberculosis Research Advisory Committee (TRAC) whose secretariat is at the Armaur Hansen Research Institute (AHRI) in Addis Ababa for providing us the opportunity and their contribution in the successful completion of the project. We would like also to thank all individuals who participated in the study including the health providers and data collectors.

We thank Dr Riitta Dlodlo, HIV program coordinator in the International Union against Tuberculosis and Lung Disease (The Union) for her unreserved assistance during the study.

\section{Funding}

This study was conducted under the Ethiopia Operational Research capacity building initiative of TRAC/FMOH supported by USAID/TB CARE I project. The Global Health Bureau, Office of Health, Infectious Disease and Nutrition (HIDN), US Agency for International Development, financially supported this study through TB CARE I under the terms of Agreement No. AID-OAA-A-10-00020. This study was made possible by the generous support of the American people through the United States Agency for International Development (USAID). The contents are the responsibility of the authors and do not necessarily reflect the views of USAID or the United States Government. The funding body has no role in the design of the study and collection, analysis, and interpretation of data and in writing the manuscript.

\section{Availability of data and materials}

The datasets supporting the conclusions of this article are included within the article and in the Additional file 1.

\section{Authors' contributions}

$\mathrm{GT}, \mathrm{BL}, \mathrm{TT}$ and $\mathrm{KT}$ contributed to the study design, data collection and data processing. GT and EK developed the draft manuscript which was finalized with the input of all authors. EK contributed to the design of the study. All authors have contributed to the interpretation of findings and revision of the manuscript. All authors have read and approved the final manuscript.

\section{Authors' information}

GT is assistant professor of clinical pharmacy in the College of Health Sciences, Mekelle University, Ethiopia; BL is a lecturer of pharmaco-epidemiology and social pharmacy in Mekelle University, College of Health Sciences, Ethiopia and Masters student in the Department of Public Health Sciences, Karolinska Institute, Sweden; $\pi T$ is tuberculosis program coordinator in Tigray regional health bureau, Ethiopia and TT is also PhD fellow student at the Department of Public Health and Clinical Medicine, Umeå University, Sweden. EK is a senior epidemiologist at KNCV Tuberculosis Foundation based in Addis Ababa, Ethiopia; and KT is assistant professor in the institute of Biomedical Sciences, College of Health Science, Mekelle University, Ethiopia.

\section{Competing interests}

The authors declare that they have no competing interests.

\section{Consent for publication}

Not applicable.

\section{Ethics approval and consent to participate}

This study was approved by the Health research Ethics Review Committee of the College of Health Sciences, Mekelle University, Ethiopia (Reference number: ERC0296/2013). Participants' records were kept confidential. Interviews and focus groups were conducted after obtaining verbal consent from each participant. Prior to obtaining consent, a written description of the study aims, benefits and risks was provided to each participant Participants were reminded of their right to withdraw from the study or terminate the interview at any time. Group consent was obtained for the focus groups and audio taping. All interviews and focus group sessions were conducted in local languages (Tigrigna and Amharic).

\section{Adherence to COREQ guidelines}

The qualitative component of our article adheres to the COREQ guidelines. A report to show the COREQ guidelines have been followed is added in this article as additional files.

\section{Author details}

'Department of Pharmacy, College of Health Sciences, Mekelle University, Mekelle, Ethiopia. ${ }^{2}$ Tuberculosis Program, Tigray Regional Health Bureau, Mekelle, Ethiopia. ${ }^{3}$ Department of Public Health Sciences, Karolinska Institute, Solna, Stockholm County, Sweden. ${ }^{4}$ Institute of Biomedical Sciences, College of Health Science, Mekelle University, Mekelle, Ethiopia. ${ }^{5} \mathrm{KNCV}$ Tuberculosis Foundation, Addis Ababa, Ethiopia. 'Department of Global Health, Academic Medical Center, University of Amsterdam, Amsterdam Institute for Global Health and Development, Amsterdam, The Netherlands.

Received: 19 December 2015 Accepted: 20 June 2016 Published online: 19 August 2016

\section{References}

1. World Health Organization. Guidelines for intensified tuberculosis casefinding and isoniazid preventive therapy for people living with HIV in resource-constrained settings. Geneva: WHO; 2011.

2. World Health Organization. Global tuberculosis report 2015. Geneva: WHO; 2015. Available at: http://www.who.int/tb/publications/global_report/en/. (Accessed 6 Dec 2015).

3. Federal Ministry of Health of Ethiopia. Implementation guideline for TB/HIV collaborative activities in Ethiopia. Addis Ababa: FMoH; 2007.

4. Woldehanna S, Volmink J. Treatment of latent tuberculosis infection in HIV infected persons. Cochrane Database Syst Rev. 2004;1:CD000171.

5. Yirdaw KD, Jerene D, Gashu Z, Edginton ME, Kumar AMV, Letamo Y, Feleke B, Teklu AM, Zewdu S, Weiss B, Ruff A. Beneficial effect of isoniazid preventive therapy and antiretroviral therapy on the incidence of tuberculosis in people living with HIV in Ethiopia. PLoS One. 2014;9(8): e104557. doi:10.1371/journal.pone.0104557.

6. Assebe LF, Reda HL, Wubeneh AD, Lerebo WT, Lambert SM. The effect of isoniazid preventive therapy on incidence of tuberculosis among HIVinfected clients under pre-ART care, Jimma, Ethiopia: a retrospective cohort study. BMC Public Health. 2015;15:346.

7. Grant AD, Charalambous S, Fielding KL, Jay KH, Corbett EL, Chaisson RE, De Cock KM, Hayes RJ, Churchyard GJ. Effect of routine isoniazid preventive therapy on tuberculosis incidence among HIV-infected men in South Africa: a novel randomized incremental recruitment study. JAMA. 2005:293:2719-25.

8. Golub JE, Pronyk P, Mohapi L, Thsabangu N, Moshabela M, Struthers H, Gray GE, McIntyre JA, Chaisson RE, Martinson NA. Isoniazid preventive therapy, HAART and tuberculosis risk in HIV-infected adults in South Africa: a prospective cohort. AIDS. 2009;23(5):631-6.

9. Zar HJ, Cotton MF, Strauss S, Karpakis J, Hussey G, Schaaf HS, Rabie H, Lombard CJ. Effect of isoniazid prophylaxis on mortality and incidence of tuberculosis in children with HIV: randomised controlled trial. BMJ. 2006; 334(7585):136

10. Bucher HC, Griffith LE, Guyatt GH, Sudre P, Naef M, Sendi P, Battegay M. Isoniazid prophylaxis for tuberculosis in HIV infection: a meta-analysis of randomized controlled trials. AIDS. 1999:13(4):501-7.

11. Akolo C, Adetifa I, Shepperd S, Volmink J. Treatment of latent tuberculosis infection in HIV infected persons. Cochrane Database Syst Rev. 2010;1: CD000171. Epub 2010 Jan 20.

12. World Health Organization. Global tuberculosis report 2013. Geneva: WHO; 2013.

13. Cowan J, Greenberg J. Assessing tuberculosis management and prevention in rural and semi-urban Ethiopia: Current practice and opportunities for improvement. University of Washington. Available at: https://depts. washington.edu/uwmedres/professional/Research/09-10/Cowan.pdf. (Accessed 2 Feb 2014)

14. Getahun H, Granich R, Sculier D, Gunneberg C, Blanc L, Nunn P, Raviglione M. Implementation of isoniazid preventive therapy for people living with HIV worldwide: barriers and solutions. AIDS. 2010;24 Suppl 5:S57-65.

15. Date AA, Vitoria M, Granich R, Banda M, Fox YM, Gilks C. Implementation of co-trimoxazole prophylaxis and isoniazid preventive therapy for people living with HIV. Bull World Health Organ. 2010;88:253-9.

16. Mindachew M, Deribew A, Memiah P, Biadgilign S. Perceived barriers to the implementation of Isoniazid preventive therapy for people living with HIV in resource constrained settings: a qualitative study. Pan Afr Med J. 2014;17:26.

17. Lester R, Hamilton R, Charalambous S, Dwadwa T, Chandler C, Churchyard GJ, Grant AD. Barriers to implementation of isoniazid preventive therapy in HIV clinics: a qualitative study. AIDS. 2010;24 Suppl 5:S45-8.

18. The government of the national state of Tigray. Report on the health status of Tigray region. August, 2014; Mekelle, Ethiopia. Available at: http:// 
aigaforum.com/documents/Peresentation_Tigray_Health_Bereau_For_ Diaspora.pdf. (Accessed 27 Sept 2014).

19. Wesen A, Mitike G. Provision and awareness for isoniazid preventive therapy among PLHIV in Addis Ababa, Ethiopia. BMC Int Health Hum Rights. 2012;12(1):2.

20. Denegetu AW, Dolamo BL. Tuberculosis case finding and isoniazid preventive therapy among people living with HIV at public health facilities of Addis Ababa, Ethiopia: a cross-sectional facility based study. BMC Public Health. 2014;14:52.

21. Legesse M, Jerene D. New tool reduces anti-TB drug stock outs in Ethiopia. 2014. April 27. Available at: https://www.msh.org/news-events/stories/newtool-reduces-anti-tb-drug-stock-outs-in-ethiopia (Accessed 8 Dec 2015).

22. Berhanemeskel E, Gedif T. Assessment of supply chain management of HIV/ AIDS related commodities in selected public hospitals and health centers in Addis Ababa, Ethiopia. Addis Ababa University electronic Thesis and dissertation June 2014. Available at: http://etd.aau.edu.et/handle/123456789/ 6099 (Accessed 8 Dec 2015).

23. Daniel G, Tegegnework H, Demissie T, Reithinger R. Pilot assessment of supply chains for pharmaceuticals and medical commodities for malaria, tuberculosis and HIV infection in Ethiopia. Trans R Soc Trop Med Hyg. 2012;106(1):60-2.

24. Mudzteba M, Gedif T. Assessment of pharmaceutical logistics system in health centers of Addis Ababa, Ethiopia. Addis Ababa University electronic Thesis and dissertation August, 2014. Available at: http://etd.aau.edu.et/ handle/123456789/6102. (Accessed 8 Dec 2015).

25. Zaeh S, Kempker R, Stenehjem E, Blumberg HM, Temesgen O, Ofotokun I, Tenna A. Improving tuberculosis screening and isoniazid preventive therapy in an HIV clinic in Addis Ababa, Ethiopia. Int J Tuberc Lung Dis. 2013;17(11):1396-401.

26. Moolphate S, Lawpoolsri S, Pungrassami P, Sanguanwongse N, Yamada N, Kaewkungwal J. Barriers to and motivations for the implementation of a treatment programme for latent tuberculosis infectionusing isoniazid for people living with HIV, in upper northern Thailand. Glob J Health Sci. 2013; 5(4):60-70. doi:10.5539/gjhs.v5n4p60.

27. Durovni B, Cavalcante SC, Saraceni V, Vellozo V, Israel G, King BS, Cohn S, Efron A, Pacheco AG, Moulton LH, Chaisson RE, Golub JE. The implementation of isoniazid preventive therapy in HIV clinics: the experience from the TB/HIV in Rio (THRio) Study. AIDS. 2010;24 Suppl 5:S49-56.

28. Balcells ME, Thomas SL, Godfrey-Faussett P, Grant AD. Isoniazid preventive therapy and risk for resistant tuberculosis. Emerg Infect Dis. 2006;12(5):744-51.

29. Family Health International 360 . Isoniazid preventive therapy for the prevention of tuberculosis in people living with HIV/AIDS. Available at: http://www.fhi360 org/sites/default/files/webpages/PT/index.html (Accessed 2 Mar 2015).

30. Van Halsema CL, Fielding KL, Chihota VN, Russell EC, Lewis JJC, Churchyard GJ, Grant AD. Tuberculosis outcomes and drug susceptibility in individuals exposed to isoniazid preventive therapy in a high HIV prevalence setting. AIDS. 2010;24(7):1051-5.

31. MRA van Cleeff, LE Kivihya-Ndugga, $\mathrm{H}$ Meme, JA Odhiambo and PR Klatser The role and performance of chest X-ray for the diagnosis of tuberculosis: A cost-effectiveness analysis in Nairobi, Kenya. BMC Infect Dis. 2005; 5:111.

32. Ait-Khaled N, Alarcon E, Bissell K, Boillot F, Caminero JA, Chiang CY, Clevenbergh P, Dlodlo R, Enarson DA, Enarson P, Ferroussier O, Fujiwara Pl, Harries AD, Heldal E, Hinderaker SG, Kim SJ, Lienhardt C, Rieder HL, Rusen ID, Trébucq A, Van Deun A, Wilson N. Isoniazid preventive therapy for people living with HIV: public health challenges and implementation issues. Int J Tuberc Lung Dis. 2009;13(8):927-35.

33. Rutherford ME, Hill PC, Triasih R, Sinfield R, van Creve R, Graham SM Preventive therapy in children exposed to Mycobacterium tuberculosis: problems and solutions. Trop Med Int Health. 2012;17(10):1264-73.

34. Antonucci G, Girardi E, Raviglione M, Vanacore P, Angarano G, Chirianni A, Pagano G. Guidelines of tuberculosis preventive therapy for HIV-infected persons: a prospective, multicentre study. Eur Respir J. 2001;18:369-75.

35. Nolan HM, Goldberg SV, Buskin SE. Hepatotoxicity associated with isoniazid preventive therapy a 7-year survey from a public health tuberculosis clinic JAMA. 1999;281(11):1014-8.

\section{Submit your next manuscript to BioMed Central and we will help you at every step:}

- We accept pre-submission inquiries

- Our selector tool helps you to find the most relevant journal

- We provide round the clock customer support

- Convenient online submission

- Thorough peer review

- Inclusion in PubMed and all major indexing services

- Maximum visibility for your research

Submit your manuscript at www.biomedcentral.com/submit
Biomed Central 